\title{
Remarks on metric transforms and fixed-point theorems
}

William A Kirk ${ }^{1}$ and Naseer Shahzad ${ }^{2 *}$

${ }^{\text {"Correspondence: }}$

nshahzad@kau.edu.sa

${ }^{2}$ Department of Mathematics, King Abdulaziz University, P.O. Box 80203

Jeddah, Saudi Arabia

Full list of author information is

available at the end of the article

\begin{abstract}
It is shown that if a mapping is a local radial contraction defined on a metric space $(X, d)$ which takes values in a metric transform of $(X, d)$, then for many metric transforms it is also a local radial contraction (with possibly different contraction constant) relative to the original metric. Several specific examples are given. This in turn implies that the mapping has a fixed point if the space is rectifiably pathwise connected. Some results about set-valued contractions are also discussed.
\end{abstract}

Keywords: metric transform; local radial contraction; rectifiably pathwise connected space; Hausdorff metric; $\mathrm{H}^{+}$-contraction; uniform local multivalued contraction; metric space

\section{Introduction}

In this paper, we study fixed points of mappings satisfying local contractive conditions, with a special emphasis on the following concept due to L. M. Blumenthal.

Definition 1.1 A strictly increasing concave function $\phi:[0, \infty) \rightarrow \mathbb{R}$ for which $\phi(0)=0$ is called a metric transform.

Blumenthal has observed (see Exercise 5 on p.26 of [1]) that if $(X, d)$ is a metric space and if $\rho(x, y)=\phi(d(x, y))$ for each $x, y \in X$, where $\phi$ is a metric transform, then $(X, \rho)$ is also a metric space. He had introduced this concept earlier in [2] to show that the metric transform $\phi(M)$ of any metric space $M$ by $\phi(t)=t^{\alpha}, 0<\alpha \leq \frac{1}{2}$, has the Euclidean four point property, i.e., each four points of $\phi(M)$ are isometric to a quadruple of points in 3-dimensional Euclidean space.

A mapping $g$ defined on a metric space $X$ is said to be a local radial contraction [3] if there exists $k \in(0,1)$ such that for each $x \in X$ there exists $\varepsilon_{x}>0$ such that $d(x, u)<\varepsilon_{x} \Rightarrow$ $d(g(x), g(u)) \leq k d(x, u)$ for all $u \in X$. We begin by showing that if a mapping is a local radial contraction defined on a metric space $(X, d)$ and taking values in a metric transform of $(X, d)$, then in many instances it is also a local radial contraction (with possibly different contraction constant) relative to the original metric. Several specific examples are given. This in turn implies that the mapping has a fixed point if the space is rectifiably pathwise connected. In Section 3, we turn our attention to set-valued contractions and prove, among other things, a set-valued analog to the main result of Section 2. Finally, because it is also based on the idea of a metric transform, we revisit a counter-example given in [4] in somewhat more detail.

๑) 2013 Kirk and Shahzad; licensee Springer. This is an Open Access article distributed under the terms of the Creative Commons Attribution License (http://creativecommons.org/licenses/by/2.0), which permits unrestricted use, distribution, and reproduction in any medium, provided the original work is properly cited. 


\section{Local radial contractions}

As noted above, a mapping $g$ defined on a metric space $X$ is a local radial contraction if there exists $k \in(0,1)$ such that for each $x \in X$ there exists $\varepsilon_{x}>0$ such that $d(x, u)<\varepsilon_{x} \Rightarrow$ $d(g(x), g(u)) \leq k d(x, u)$ for all $u \in X$. If this condition is satisfied for some $\varepsilon>0$ independent of $x$ then $g$ is said to be a uniform local contraction.

The following is the central result of [4]. Recall that a rectifiable path is a path of finite length (see, e.g., [5, p.34]).

Theorem 2.1 ([4]) Let $(X, d)$ be a complete metric space, and suppose each two points of $X$ can be joined by a rectifiable path. Then if $g: X \rightarrow X$ is a local radial contraction, $g$ has a unique fixed point $x_{0} \in X$, and moreover $\lim _{n \rightarrow \infty} g^{n}(x)=x_{0}$ for each $x \in X$.

Rakotch proved the above theorem in [6] under the stronger assumption that $g$ is a local contraction in the sense that there exists $k \in(0,1)$ such that each point of $x \in X$ has a neighborhood $N_{x}$ such that $d(g(u), g(v)) \leq k d(u, v)$ for all $u, v \in N_{x}$. The proof of Theorem 2.1 entails showing that the metric space $(X, \ell)$ is complete, where $\ell$ is the path metric on $X$ induced by $d$, and then observing that $g$ is actually a global contraction on $(X, \ell)$. (An assertion in the proof of Theorem 2.1 given in [4] was based on a Proposition of Holmes [3], which was later shown by Jungck [7] to be false. However, as Jungck also proved in [7], the assertion itself is true. Hence, the proof given in [4], with minor changes, is true.)

We now give a simple condition in terms of metric transforms which implies that a mapping $g: X \rightarrow X$ is a local radial contraction. Notice that if $\phi$ is taken to be the identity mapping, the following result reduces to the definition of a local radial contraction.

Theorem 2.2 Let $(X, d)$ be a metric space and $g: X \rightarrow X$. Suppose there exists a metric transform $\phi$ on $X$ and a number $k \in(0,1)$ such that the following conditions hold:

(a) For each $x \in X$ there exists $\varepsilon_{x}>0$ such that $d(x, u)<\varepsilon_{x} \Rightarrow$

$$
\phi(d(g(x), g(u))) \leq k d(x, u) .
$$

(b) There exists $c \in(0,1)$ such that for all $t>0$ sufficiently small

$$
k t \leq \phi(c t) .
$$

Then $g$ is a local radial contraction on $(X, d)$.

In view of Theorem 2.1, we now have the following.

Theorem 2.3 Suppose, in addition to the assumptions in Theorem 2.2, $X$ is complete and rectifiably pathwise connected. Then $g$ has a unique fixed point $x_{0}$, and $\lim _{n \rightarrow \infty} g^{n}(x)=x_{0}$ for each $x \in X$.

Proof of Theorem 2.2 Let $x \in X$. Then if $d(x, u)<\varepsilon_{x}$,

$$
\phi(d(g(x), g(u))) \leq k d(x, u) .
$$


Now suppose there exists $c \in(0,1)$ such that for $t$ sufficiently small,

$$
k t \leq \phi(c t) .
$$

This implies there exists $\delta_{x}>0$ with $\delta_{x} \leq \varepsilon_{x}$ such that $d(x, u)<\delta_{x} \Rightarrow$

$$
\phi(d(g(x), g(u))) \leq k d(x, u) \leq \phi(c d(x, u)) .
$$

Since $\phi$ is strictly increasing, $d(x, u)<\delta_{x} \Rightarrow$

$$
d(g(x), g(u)) \leq c d(x, u) .
$$

Therefore, $g$ is a local radial contraction on $(X, d)$.

Remark 2.4 If condition (a) is changed to

$$
\phi(d(g(x), g(y))) \leq k d(x, y) \quad \text { for all } x, y \in X,
$$

then $g$ is a uniform local contraction on $(X, d)$. This is because condition (b) now implies that there exists $\delta>0$ such that $d(x, y)<\delta \Rightarrow$

$$
\phi(d(g(x), g(y))) \leq k d(x, y) \leq \phi(c d(x, y)) .
$$

Remark 2.5 If $g: X \rightarrow X$ is onto and satisfies the following expansive type condition: there exists $k \in(0,1)$ such that

$$
d(g(x), g(y)) \geq k^{-1} \phi(d(x, y)) \quad \text { for all } x, y \in X,
$$

then $g^{-1}$ is a uniform local contraction on $(X, d)$. This is because $g^{-1}$ exists and satisfies

$$
\phi\left(d\left(g^{-1}(x), g^{-1}(y)\right)\right) \leq k d(x, y) \quad \text { for all } x, y \in X .
$$

Condition (b) might appear to be too restrictive. We now list several examples of nontrivial metric transforms for which the condition holds.

(i) $\phi(t)=\frac{t}{1+t}$. Let $k \in(0,1)$ and select $c \in(k, 1)$. Then

$$
k t \leq \phi(c t) \quad \Leftrightarrow \quad t \leq \frac{\frac{c t}{1+c t}}{k} \quad \Leftrightarrow \quad k t \leq \frac{c t}{1+c t} \quad \Leftrightarrow \quad k \leq \frac{c}{1+c t} \quad \Leftrightarrow \quad t \leq \frac{c-k}{c k} .
$$

Since $c>k$, condition (b) follows.

(ii) $\phi(t)=t^{\beta}$, for $\beta \in(0,1)$. Then for any $c, k \in(0,1)$

$$
t \leq \frac{\phi(c t)}{k} \Leftrightarrow t \leq \frac{(c t)^{\beta}}{k}
$$

and condition (b) holds for $t \leq 1$. 
(iii) $\phi(t)=\sin \left(\frac{t}{1+t}\right)$. Let $k \in(0,1)$, and set $h(t)=\frac{t}{1+t}$. We know that if $c \in(k, 1)$ and if $t \leq \frac{c-k}{c k}$ then

$$
k t \leq h(c t)
$$

In particular, take $k^{\prime} \in(k, 1)$, then choose $c \in\left(k^{\prime}, 1\right)$. The same argument as in (ii) shows that if $t \leq \frac{c-k^{\prime}}{c k^{\prime}}$ then

$$
k t<k^{\prime} t \leq h(c t)
$$

Thus, if $t$ is sufficiently small,

$$
k t \leq \sin k^{\prime} t \leq \sin (h(c t))=\phi(c t) .
$$

(iv) $\phi(t)=p \tan ^{-1} t$ for fixed $p>1$. Let $k \in(0,1)$. Then $k t \leq \phi(c t) \Leftrightarrow \tan \left(\frac{k t}{p}\right) \leq c t$. Let $f(t)=$ $c t-\tan \left(\frac{k t}{p}\right)$. Then $f(0)=0$ and $f^{\prime}(t)=c-\frac{k}{p} \sec ^{2}\left(\frac{k t}{p}\right)>0 \Leftrightarrow \sec ^{2}\left(\frac{k t}{p}\right)<\frac{p c}{k}$. If $c \in(0,1)$ is chosen so that $\frac{p c}{k}>1$, then $f^{\prime}(t)>0$ for $t>0$ sufficiently small. This implies that $f(t)>0$ for $t>0$ sufficiently small, and this in turn implies that condition (b) holds.

(v) $\phi(t)=\ln (1+t)$. Let $k \in(0,1)$ and select $c \in(k, 1)$. Then $k t \leq \phi(c t) \Leftrightarrow e^{k t} \leq 1+c t$. Let $f(t)=1+c t-e^{k t}$. Then $f(0)=0$ and for $t>0, f^{\prime}(t)>0 \Leftrightarrow e^{k t}<\frac{c}{k} \Leftrightarrow t<k^{-1} \ln \left(\frac{c}{k}\right)$. This is clearly true for $t>0$ sufficiently small because $c \in(k, 1)$.

Not every metric transform satisfies condition (b); $\phi(t)=\tan ^{-1} t$ provides an example. On the other hand, Proposition 2.6 below shows that the collection of metric transforms which do satisfy condition (b) are indeed numerous and complex.

Proposition 2.6 Let $\mathfrak{M}$ denote the class of all metric transforms $\phi$ with the property that $\phi$ is twice differentiable, and let $\mathfrak{M}_{1}$ denote the subfamily of $\mathfrak{M}$ consisting of those $\phi \in \mathfrak{M}$ which satisfy the following condition: for any $k \in(0,1)$ there exists $c \in(0,1)$ such that for $t>0$ sufficiently small,

$$
k t \leq \phi(c t) .
$$

Then both $\mathfrak{M}$ and $\mathfrak{M}_{1}$ are closed under functional composition.

Proof Let $\phi, \psi \in \mathfrak{M}$ and let $\varphi=\phi \circ \psi$. Then $\varphi(0)=\phi \circ \psi(0)=0$. Also for any $t>0$,

$$
\varphi^{\prime}(t)=\phi^{\prime}(\psi(t)) \cdot \psi^{\prime}(t)>0
$$

and

$$
\varphi^{\prime \prime}(t)=\phi^{\prime}(\psi(t)) \cdot \psi^{\prime \prime}(t)+\phi^{\prime \prime}(\psi(t)) \cdot\left[\psi^{\prime}(t)\right]^{2}<0 .
$$

Therefore, $\varphi \in \mathfrak{M}$.

Now suppose $\phi, \psi \in \mathfrak{M}_{1}$. Then there exists $c_{1} \in(0,1)$ such that for $t>0$ sufficiently small,

$$
k t \leq \phi\left(c_{1} t\right) .
$$


Also, there exists $c \in(0,1)$ such that for $t>0$ sufficiently small

$$
c_{1} t \leq \psi(c t)
$$

Since $\phi$ is strictly increasing,

$$
c_{1} t \leq \psi(c t) \quad \Leftrightarrow \quad \phi\left(c_{1} t\right) \leq \phi(\psi(c t)) .
$$

Therefore, $k t \leq \varphi(c t)$ for $t>0$ sufficiently small, so it follows that $\varphi \in \mathfrak{M}_{1}$.

Finally, we observe that in Theorem 2.1 it needs only be assumed that some iterate of the mapping $g$ is a local radial contraction. Specifically, we have the following.

Theorem 2.7 Let $X$ be a complete metric space for which each two points can be joined by a rectifiable path, and suppose $g: X \rightarrow X$ is a mapping for which $g^{N}$ is a local radial contraction for some $N \in \mathbb{N}$. Then $g$ has a unique fixed point $x_{0}$, and $\lim _{n \rightarrow \infty} g^{n}(x)=x_{0}$ for each $x \in X$.

Notice that $g$ is not even assumed to be continuous. Similarly, we also have the following extension of Theorem 2.3.

Theorem 2.8 Let $X$ be a complete metric space for which each two points can be joined by a rectifiable path, and suppose $g: X \rightarrow X$ is a mapping for which $g^{N}$ satisfies the assumptions in Theorem 2.2 for some $N \in \mathbb{N}$. Then $g$ has a unique fixed point $x_{0}$, and $\lim _{n \rightarrow \infty} g^{n}(x)=x_{0}$ for each $x \in X$.

These results are immediate consequences of Theorems 2.1 and 2.3, and the following proposition due to Tan [8].

Theorem 2.9 Let $X$ be a topological space, let $x_{0} \in X$, and let $g: X \rightarrow X$ be a mapping for which $f:=g^{N}$ satisfies $\lim _{n \rightarrow \infty} f^{n}(x)=x_{0}$ for each $x \in X$. Then $\lim _{n \rightarrow \infty} g^{n}(x)=x_{0}$ for each $x \in X$. (Also if $x_{0}$ is the unique fixed point of $f$, it is also the unique fixed point of $g$.)

Holmes makes the following claim in [3]. If $(X, d)$ is a connected and locally connected metric space and $g: X \rightarrow X$ is a uniformly continuous local radial contraction, then there exists a metric $\delta$ on $X$, topologically equivalent to $d$, such that $g$ is a global contraction on $(X, \delta)$. If true, this claim would yield Theorem 2.7 for uniformly continuous local radial contractions in a more general class of spaces. However, we append an example, taken from [4], which shows that this assertion is false. We do not know if it is true when $(X, d)$ is rectifiably pathwise connected.

\section{Set valued contractions}

Let $(X, d)$ be a metric space and let $\mathcal{C B}(X)$ denote the family of nonempty, closed and bounded subsets of $X$. For $A, B \in \mathcal{C B}(X)$ let $\rho(A, B)=\sup _{x \in A} \operatorname{dist}(x, B)$ and $\rho(B, A)=$ $\sup _{x \in B} \operatorname{dist}(x, A)$. The usual Hausdorff distance $H(A, B)$ between $A$ and $B$ is defined as

$$
H(A, B)=\max \{\rho(A, B), \rho(B, A)\} .
$$


A mapping $T: X \rightarrow \mathcal{C B}(X)$ is called a multivalued contraction mapping if there exists a constant $k \in(0,1)$ such that

$$
H(T x, T y) \leq k d(x, y), \quad x, y \in X .
$$

A point $x \in X$ is said to be a fixed point of $T$ if $x \in T x$. Our point of departure in this section is the following celebrated theorem of Nadler [9].

Theorem 3.1 Let $(X, d)$ be a complete metric space, and suppose $T: X \rightarrow \mathcal{C B}(X)$ be a multivalued contraction mapping. Then $T$ has a fixed point.

Our purpose in this section is to extend Nadler's theorem by replacing the Hausdorff metric with other metrics on $\mathcal{C B}(X)$ which are either metrically or sequentially equivalent to $H$.

One example of a metric on $\mathcal{C B}(X)$ which is metrically equivalent to the Hausdorff metric $H$ is the metric $H^{+}$, which was introduced in [10]. $H^{+}$is defined by setting

$$
H^{+}(A, B)=\frac{1}{2}(\rho(A, B)+\rho(B, A)), \quad A, B \in \mathcal{C B}(X) .
$$

Clearly, $H^{+}$is metrically equivalent to the Hausdorff metric:

$$
\frac{1}{2} H(A, B) \leq H^{+}(A, B) \leq H(A, B) .
$$

A multivalued mapping $T: X \rightarrow \mathcal{C B}(X)$ is called an $H^{+}$-contraction if

(1) there exists $k \in(0,1)$ such that

$$
H^{+}(T x, T y) \leq k d(x, y) \quad \text { for every } x, y \in X
$$

and

(2) for every $x \in X$ and $y \in T x$,

$$
\operatorname{dist}(y, T y) \leq H^{+}(T x, T y)
$$

It follows immediately from the definition of the Hausdorff metric $H$ that if $A, B \in \mathcal{C B}(X)$ and if $x \in X$. Then for each $\varepsilon>0$ there exists $y \in Y$ such that

$$
d(x, y) \leq H(A, B)+\varepsilon .
$$

Thus, condition (2) is always true if $H^{+}$is replaced with the usual Hausdorff metric $H$. This is precisely the fact about the Hausdorff metric that Nadler used in his proof. In fact, Nadler's proof yields the following result. This theorem implies that every $\mathrm{H}^{+}$-contraction of a complete metric space $X$ into $\mathcal{C B}(X)$ has a fixed point [10].

Theorem 3.2 Let $(X, d)$ be a complete metric space, and let $D$ be any metric on $\mathcal{C B}(X)$ which is sequentially equivalent to the Hausdorff metric $H$. Suppose $T: X \rightarrow \mathcal{C B}(X)$ satisfies 
(1) there exists $k \in(0,1)$ such that

$$
D(T x, T y) \leq k d(x, y) \quad \text { for every } x, y \in X
$$

and

(2) if $x \in X$ and $y \in T x$,

$$
\operatorname{dist}(y, T y) \leq D(T y, T x) .
$$

Then $T$ has a fixed point, i.e., there exists $x \in X$ such that $x \in T x$.

Proof (cf. [9]) By saying $D$ is sequentially equivalent to $H$, we mean that for $A \in \mathcal{C B}(X)$ and $\left\{A_{n}\right\} \subset \mathcal{C B}(X)$,

$$
\lim _{n \rightarrow \infty} D\left(A_{n}, A\right)=0 \quad \Leftrightarrow \quad \lim _{n \rightarrow \infty} H\left(A_{n}, A\right)=0
$$

Suppose $T: X \rightarrow \mathcal{C B}(X)$. Select $x_{0} \in X$ and $x_{1} \in T x$. By (2) and (1), there exists $x_{2} \in T x_{1}$ such that

$$
\begin{aligned}
d\left(x_{1}, x_{2}\right) & \leq D\left(T x_{0}, T x_{1}\right)+k \\
& \leq k d\left(x_{0}, x_{1}\right)+k .
\end{aligned}
$$

Similarly, there exists $x_{3} \in T x_{2}$ such that

$$
\begin{aligned}
d\left(x_{2}, x_{3}\right) & \leq D\left(T x_{1}, T x_{2}\right)+k^{2} \\
& \leq k d\left(x_{1}, x_{2}\right)+k^{2} \\
& \leq k\left[k d\left(x_{0}, x_{1}\right)+k\right]+k^{2} \\
& =k^{2} d\left(x_{0}, x_{1}\right)+2 k^{2} .
\end{aligned}
$$

In general, for each $i \in \mathbb{N}$ there exists $x_{i+1} \in T x_{i}$ such that

$$
\begin{aligned}
d\left(x_{i}, x_{i+1}\right) & \leq D\left(T x_{i-1}, x_{i}\right)+k^{i} \\
& \leq k d\left(x_{i-1}, x_{i}\right)+k^{i} \\
& \leq k\left[D\left(T x_{i-2}, T x_{i-1}\right)+k^{i-1}\right]+k^{i} \\
& \leq k^{2} d\left(x_{i-2}, x_{i-1}\right)+2 k^{i} \\
& \leq \cdots \\
& \leq k^{i} d\left(x_{0}, x_{1}\right)+i k^{i} .
\end{aligned}
$$

Therefore,

$$
\sum_{i=0}^{\infty} d\left(x_{i}, x_{i+1}\right) \leq d\left(x_{0}, x_{1}\right) \sum_{i=0}^{\infty} k^{i}+\sum_{i=0}^{\infty} i k^{i}<\infty
$$


Hence, $\left\{x_{n}\right\}$ is a Cauchy sequence, so there exists $x \in X$ such that $\lim _{n \rightarrow \infty} x_{n}=x$. It follows from (1) that $\lim _{n \rightarrow \infty} D\left(T x_{n}, T x\right)=0$. Since $D$ and $H$ are equivalent, $\lim _{n \rightarrow \infty} H\left(T x_{n}, T x\right)=0$. Since $x_{n+1} \in T x_{n}$, it follows from the definition of Hausdorff metric that $\lim _{n \rightarrow \infty} \operatorname{dist}\left(x_{n}\right.$, $T x)=0$, and since $T x$ is closed, $x \in T x$.

Remark 3.3 The point valued analog of Theorem 3.2 is rather trivial. Let $(X, d)$ be a complete metric space, and let $\rho$ be any metric on $X$ which is sequentially equivalent to the $d$. Suppose $T: X \rightarrow X$ satisfies

(1) there exists $k \in(0,1)$ such that

$$
\rho(T x, T y) \leq k d(x, y) \quad \text { for every } x, y \in X
$$

and

(2) if $x \in X$,

$$
d\left(T x, T^{2} x\right) \leq \rho\left(T x, T^{2} x\right) .
$$

Then $T$ has a fixed point.

Proof Combining (1) and (2), we have

$$
d\left(T x, T^{2} x\right) \leq k d(x, T x), \quad x \in X
$$

This implies that $\left(T^{n} x\right)$ is a Cauchy sequence in $(X, d)$, so $\lim _{n \rightarrow \infty} T^{n} x=x_{0}$ exists. Since (1) implies $T$ is continuous, $T x_{0}=x_{0}$.

As noted above, (1) alone is sufficient if $D=H$ because (2) is redundant in this case. However, the following example shows that (1) alone is not sufficient if $D=H^{+}$.

Example Take $X=[0, \infty)$ with the metric: $d(x, y)=\frac{|x-y|}{|x-y|+1} \forall x, y \in X$. Define $T: X \rightarrow$ $\mathcal{C B}(X)$ by setting $T(x)=[x+1, \infty)$. Clearly, $T$ has no fixed point. However, if $x>y$ then $T(x) \subseteq T(y)$, and

$$
\begin{aligned}
H^{+}(T(x), T(y)) & =\frac{1}{2} d(T(x), T(y)) \\
& =\frac{1}{2} \frac{|x+1-(y+1)|}{|x+1-(y+1)|+1} \\
& =\frac{1}{2} \frac{|x-y|}{|x-y|+1} \\
& =\frac{1}{2} d(x, y) .
\end{aligned}
$$

We now turn to an analog of Theorem 2.2 for set-valued mappings.

A mapping $T: X \rightarrow \mathcal{C B}(X)$ is said to be an $(\varepsilon, k)$-uniform local multi-valued contraction (where $\varepsilon>0$ and $k \in(0,1)$ ) if for $x, y \in X, d(x, y)<\varepsilon \Rightarrow H(T x, T y) \leq k d(x, y)$. This definition, given in [9], is modelled after a concept introduced by Edelstein in [11]. 
Theorem 3.4 Let $(X, d)$ be a metric space and $T: X \rightarrow \mathcal{C B}(X)$. Suppose there exists a metric transform $\phi$ and $k \in(0,1)$ such that the following conditions hold:

(a) For each $x, y \in X$,

$$
\phi(H(T x, T y)) \leq k d(x, y) .
$$

(b) There exists $c \in(0,1)$ such that for $t>0$ sufficiently small,

$$
k t \leq \phi(c t) .
$$

Then for $\varepsilon>0$ sufficiently small, $T$ is an $(\varepsilon, c)$-uniform local multivalued contraction on $(X, d)$.

Proof Let $x, y \in X$, and observe that

$$
\phi(H(T x, T y)) \leq k d(x, y)
$$

Now suppose there exists $c \in(0,1)$ such that for $t$ sufficiently small,

$$
k t \leq \phi(c t) .
$$

Then for $d(x, y)$ sufficiently small,

$$
\phi(H(T x, T y)) \leq k d(x, y) \leq \phi(c d(x, y))
$$

and since $\phi$ is strictly increasing this in turn implies

$$
H(T x, T y) \leq c d(x, y)
$$

Thus, for $\varepsilon>0$ sufficiently small, $T$ is an $(\varepsilon, c)$-uniform local multivalued contraction on $(X, d)$.

A metric space $(X, d)$ is said to be $\varepsilon$-chainable (where $\varepsilon>0$ is fixed) if given $a, b \in X$ there is an $\varepsilon$-chain joining $a$ and $b$. This means there exists a finite set of points $\left\{x_{i}\right\}_{i=1}^{n}$ in $X$ such that $a=x_{1}, b=x_{n}$ and $d\left(x_{i}, x_{i+1}\right)<\varepsilon$ for all $i=1, \ldots, n-1$. The following result is also due to Nadler.

Theorem 3.5 ([9, Theorem 6]) Let $(X, d)$ be a complete $\varepsilon$-chainable metric space. If $T$ : $X \rightarrow \mathcal{C B}(X)$ is an $(\varepsilon, k)$ - uniform local multivalued contraction, then $T$ has a fixed point.

By combining the above result with Theorem 3.4 we obtain the following.

Theorem 3.6 If, in addition to the assumptions of Theorem 3.4, $X$ is complete and connected, then $T$ has a fixed point.

Proof A connected metric space is $\varepsilon$-chainable for any $\varepsilon>0$. 


\section{Appendix}

The following example was given in [4]. It shows that Theorem 2.3 is false if the space is merely assumed to be pathwise connected rather than rectifiably pathwise connected. This illustrates another application of the idea of metric transforms.

Example Let $\left(\beta_{n}\right)_{n=-\infty}^{\infty}$ be a strictly increasing doubly infinite sequence in $(0,1)$. For $x, y \in$ $[0, \infty), x \leq y$, set

$$
\rho(x, y)=\left\{\begin{array}{l}
|x-y|^{\beta_{n}} \quad \text { if } x, y \in[n, n+1], \\
|x-(n+1)|^{\beta_{n}}+(p-1)+|(n+p)-y|^{\beta_{n+p}} \\
\quad \text { if } x \in[n, n+1], y \in[n+p, n+p+1], p \in \mathbb{N} .
\end{array}\right.
$$

We first observe that $\left(\mathbb{R}^{1}, \rho\right)$ is a metric space (see Proposition 4.1 below).

Now define $g: \mathbb{R}^{1} \rightarrow \mathbb{R}^{1}$ by setting $g(x)=x+1$. This mapping is a homeomorphism which is a local contraction for any $k \in(0,1)$. To see this, suppose $x, y \in[n, n+1]$. Then

$$
\rho(g(x), g(y))=|x-y|^{\beta_{n+1}} \leq k|x-y|^{\beta_{n}}=k \rho(x, y)
$$

if and only if $|x-y|^{\beta_{n+1}-\beta_{n}} \leq k$. Since $\beta_{n+1}-\beta_{n}>0$, this is always true if $|x-y|$ is sufficiently small; indeed

$$
\rho(x, y)=|x-y|^{\beta_{n}} \leq k^{\beta_{n} /\left(\beta_{n+1}-\beta_{n}\right)} \quad \Leftrightarrow \quad|x-y|^{\beta_{n+1}-\beta_{n}} \leq k .
$$

To deal with the case $x=n>0$, merely take a neighborhood of $x$ with radius less than $\min \left\{k^{\beta_{n} /\left(\beta_{n+1}-\beta_{n}\right)}, k^{\beta_{n+1} /\left(\beta_{n+2}-\beta_{n+1}\right)}\right\}$.

Notice that the mapping of the above example is even locally contractive in the sense of Rakotch [6], but it is fixed-point-free. We note also that the space $\left(\mathbb{R}^{1}, \rho\right)$ is topologically equivalent to $\mathbb{R}^{1}$ with its usual metric. In particular $\left(\mathbb{R}^{1}, \rho\right)$ is complete, connected, and locally connected. (A space $X$ is said to be locally connected if given any $x \in X$, each neighborhood $U$ of $x$ contains a connected neighborhood $V$ of $x$.)

The technique of the example is a special case of 'gluing' of metric spaces (see, e.g., [12, p.67]). Specifically, we use the following fact, which is a special case of Lemma 5.34 of [12].

Proposition 4.1 Suppose $\left(M_{1}, d_{1}\right)$ and $\left(M_{2}, d_{2}\right)$ are metric spaces with $M_{1} \cap M_{2}=\{u\}$. For $x, y \in X:=M_{1} \cup M_{2}$ set

$$
\begin{aligned}
& \rho(x, y)=d_{i}(x, y) \quad \text { if } x, y \in M_{i}, i=1,2 ; \\
& \rho(x, y)=d_{1}(x, u)+d_{2}(u, y) \quad \text { if } x \in M_{1}, y \in M_{2} .
\end{aligned}
$$

Then $(X, \rho)$ is a metric space.

We now observe that for each $n \in \mathbb{Z}$ and $\beta_{n} \in(0,1)$, the metric transform $\phi_{n}(t)=t^{\beta_{n}}$ induces a metric on the interval $[n, n+1]$. The metric space $\left(\mathbb{R}^{1}, \rho\right)$ is obtained by simply 'gluing' the consecutive intervals at their common endpoints and applying Proposition 4.1 inductively. This results in the metric defined by (1). 


\section{Competing interests}

The authors declare that they have no competing interests.

\section{Authors' contributions}

All authors contributed equally and significantly in writing this paper. All authors read and approved the final manuscript.

\section{Author details}

${ }^{1}$ Department of Mathematics, University of lowa, lowa, 52242, USA. ${ }^{2}$ Department of Mathematics, King Abdulaziz University, P.O. Box 80203, Jeddah, Saudi Arabia.

\section{Acknowledgements}

The authors are grateful to the anonymous referees for their comments that considerably improved the paper. The research of N. Shahzad was partially supported by the Deanship of Scientific Research (DSR), King Abdulaziz University, Jeddah, Saudi Arabia.

Received: 19 October 2012 Accepted: 2 April 2013 Published: 22 April 2013

\section{References}

1. Blumenthal, LM: Theory and Applications of Distance Geometry, 2nd edn. Chelsea, New York (1970)

2. Blumenthal, LM: Remarks concerning the Euclidean four-point property. Ergebnisse Math. Kolloq. Wien 7, 7-10 (1936)

3. Holmes, RD: Fixed points for local radial contractions. In: Swaminathan, S (ed.) Fixed Point Theory and Its Applications, pp. 79-89. Academic Press, New York (1976)

4. Hu, T, Kirk, WA: Local contractions in metric spaces. Proc. Am. Math. Soc. 68, 121-124 (1978)

5. Burago, D, Burago, Y, Ivanov, S: A Course in Metric Geometry. Graduate Studies in Mathematics, vol. 33. Am. Math. Soc., Providence (2001)

6. Rakotch, E: A note on $\alpha$-locally contractive mappings. Bull. Res. Counc. Isr. 40, 188-191 (1962)

7. Jungck, G: Local radial contractions - a counter-example. Houst. J. Math. 8, 501-506 (1982)

8. Tan, KK: Fixed point theorems for nonexpansive mappings. Pac. J. Math. 41, 829-842 (1972)

9. Nadler, SB Jr.: Multi-valued contraction mappings. Pac. J. Math. 30, 475-488 (1969)

10. Pathak, HK, Shahzad, N: A generalization of Nadler's fixed point theorem and its application to nonconvex integral inclusions. Topol. Methods Nonlinear Anal. 41, 207-227 (2013)

11. Edelstein, M: An extension of Banach's contraction principle. Proc. Am. Math. Soc. 12, 7-10 (1961)

12. Bridson, M, Haefliger, A: Metric Spaces of Non-Positive Curvature. Springer, Berlin (1999)

doi:10.1186/1687-1812-2013-106

Cite this article as: Kirk and Shahzad: Remarks on metric transforms and fixed-point theorems. Fixed Point Theory and Applications 2013 2013:106.

\section{Submit your manuscript to a SpringerOpen ${ }^{\ominus}$ journal and benefit from:}

- Convenient online submission

- Rigorous peer review

Immediate publication on acceptance

- Open access: articles freely available online

- High visibility within the field

- Retaining the copyright to your article 